\title{
When tonic cardiac vagal tone predicts changes in phasic vagal tone: The role of fear and perceptual load
}

\section{GEWNHI PARK, ${ }^{a}$ MICHAEL W. VASEY, ${ }^{\mathrm{b}}$ JAY J. VAN BAVEL, ${ }^{\mathrm{c}}$ AND JULIAN F. THAYER ${ }^{\mathrm{b}}$}

a Department of Psychology, Azusa Pacific University, Azusa, California, USA

${ }^{b}$ Department of Psychology, The Ohio State University, Columbus, Ohio, USA

'Department of Psychology, New York University, New York, New York, USA

\begin{abstract}
We examined the relationship between tonic — a correlate of self-regulatory functioning — and phasic cardiac vagal activity (indexed by heart rate variability; HRV) during a selective attentional task with varying levels of load. Participants detected a target letter among letter strings superimposed on either fearful or neutral face distractors. Letter strings consisted of six target letters under low load and one target letter and five nontarget letters under high load. With fearful distractors, lower tonic HRV was associated with phasic HRV suppression, suggesting an autonomic stress response under both low and high load. In contrast, higher tonic HRV was associated with phasic HRV enhancement, suggesting greater self-regulatory effort under low load and an absence of phasic HRV suppression under high load. The current research suggests that tonic cardiac vagal tone is associated with the ability to flexibly adapt autonomic responses.
\end{abstract}

Descriptors: Cardiac vagal tone, Self-regulatory effort

Self-regulation refers to the ability to control one's thoughts, emotions, and behavior, thereby enabling an organism to select optimal responses to meet situational demands (Gross 1998; Segerstrom \& Nes, 2007; Thayer \& Lane, 2000). Self-regulatory capacity has a profound impact on a wide spectrum of human life (Appelhans \& Luecken, 2006; Eisenberg, 2001; Oveis et al., 2009). Children with good self-regulatory capacity are less likely to express frustration, irritation, and aggression, and are more likely to get higher grades in school, compared to children with poor self-regulatory capacity (Funder \& Block, 1989; Funder, Block, \& Block, 1983). People with poor self-regulation are more likely to use alcohol and drugs and to engage in criminal and delinquent behavior (Gottfredson \& Hirschi, 1990; McCullough \& Willoughby, 2009). Similarly, the inability to self-regulate is an important characteristic of many forms of psychopathology, including depression (Pyszczynski, Holt, \& Greenberg, 1987; Wenzlaff, Wegner, \& Roper, 1988), obsessive or ruminative thoughts (Martin \& Tesser, 1989; Wegner, Schneider, Carter, \& White, 1987), and aggression (Gottfredson \& Hirschi, 1990; Tice \& Baumeister, 1993). The current study examines whether tonic cardiac vagal tone-a correlate of selfregulatory functioning-is associated with changes in phasic cardiac activity reflecting the exertion of self-regulatory effort during an implicit emotion regulation task with varying levels of cognitive load.

Address correspondence to: Gewnhi Park, Department of Psychology, Azusa Pacific University, 901 E. Alosta Ave., Azusa, CA 91702, USA. E-mail: flyingbluesky.park@gmail.com

\section{The Neurovisceral Integration Model and Heart Rate Variability}

A number of studies have identified a link between self-regulatory functioning and resting or tonic cardiac vagal activity (Appelhans \& Luecken, 2006; Park, Vasey, Van Bavel, \& Thayer, 2013; Porges, 2003; Thayer \& Lane, 2000). Effective self-regulation is associated with robust cardiac vagal control, which contributes to adaptive and flexible responses to meet various situational demands (Berntson et al., 1997; Park, Vasey et al., 2013; Task Force of the European Society of Cardiology and the North American Society of Pacing and Electrophysiology, 1996; Thayer \& Lane, 2000). Moreover, the neural circuits responsible for self-regulatory functioning are also involved in cardiac vagal control (Berntson et al., 1997; Park, Vasey et al., 2013; Task Force, 1996; Thayer, Hansen, Saus-Rose, \& Johnsen, 2009; Thayer \& Lane, 2000). High vagally mediated tonic heart rate variability (HRV) — a widely used measure of cardiac vagal tone (Berntson et al., 1997; Task Force, 1996; Thayer \& Lane, 2000) - is associated with cognitive, emotional, and autonomic self-regulatory capacity (Thayer et al., 2009, 2000). For example, people with high tonic HRV cope better with stress (Brosschot, Van Dijk, \& Thayer, 2007), express greater positive emotion (Fabes \& Eisenberg, 1997; Oveis et al., 2009), and make faster and more accurate responses in cognitive tasks that assess executive function (Hansen, Johnsen, \& Thayer, 2003; Park, Van Bavel, Vasey, \& Thayer, 2012; Thayer et al., 2009). In contrast, low tonic HRV is associated with poor self-regulatory capacity, including rigid, hypervigilant responses (Thayer et al., 2009; Thayer \& Lane, 2000). In the current study, we examine whether tonic 
cardiac vagal activity is associated with phasic cardiac vagal activity, which reflects the exertion of self-regulatory effort, in response to emotional stimuli under load.

\section{Phasic Changes in HRV and Self-Regulatory Effort}

Compared to tonic cardiac vagal activity, relatively little is known about the role of phasic cardiac activity in the context of selfregulation (Butler, Wilhelm, \& Gross, 2006; Ingjaldsson, Laberg, \& Thayer, 2003; Segerstrom \& Nes, 2007; Smith et al., 2011). Reduced phasic cardiac activity, or vagal suppression, has long been construed as an autonomic response to stress (Beauchaine, Gatzke-Kopp, \& Mead, 2007; Beauchaine, Katkin, Strassberg, \& Snarr, 2001; El-Sheikh, Hinnant, \& Erath, 2011; Schwerdtfeger \& Derakhshan, 2010). When people are directly exposed to a stressor, such as a video clip depicting an escalating conflict between peers (Beauchaine et al., 2001, 2007; El-Sheikh et al., 2011), performing a difficult mental stress task (Weber et al., 2010), or engaging in a reaction time shock avoidance task in which a shock is threatened as a penalty for slow performance (Uijtdehaage \& Thayer, 2000), phasic HRV suppression occurs. Furthermore, phasic HRV suppression is observed when people are engaged in worry and fear or anger imagery (Lyonfields, Borkovec, \& Thayer, 1995; Thayer, Friedman, \& Borkovec, 1996). As such, phasic HRV suppression has been conceptualized as a physiological response that represents the withdrawal of cardiac vagal control and the activation of the defensive systems (Thayer et al., 1996). These studies find a positive association between higher tonic HRV and greater phasic HRV suppression to stress, which suggests that phasic HRV may reflect emotional regulation and serves a protective function against environment challenges (Beauchaine et al., 2001, 2007; El-Sheikh et al., 2011). Importantly, when the stressor is over, people with higher tonic HRV show increased phasic HRV during the recovery (Weber et al., 2010). In contrast, lower tonic HRV is associated with reduced phasic HRV suppression to stress, which may increase the risk of developing behavioral or emotional problems (Appelhans \& Luecken, 2006; Beauchaine et al., 2001, 2007; El-Sheikh et al., 2011).

Another line of research has provided evidence that increased phasic cardiac activity is associated with the successful exertion of self-regulatory effort (Ingjaldsson et al., 2003; Lane et al., 2013; Segerstrom \& Nes, 2007; Smith et al., 2011; Thayer \& Lane, 2009). For example, alcoholics with good impulse control over drinking showed higher phasic HRV while exposed to alcoholic cues (Ingjaldsson et al., 2003). Moreover, there is some evidence suggesting that tonic cardiac vagal activity modulates phasic cardiac vagal activity indicative of self-regulatory effort. For example, people showed higher phasic HRV when instructed to eat only carrots, which required greater self-regulatory effort, compared to when instructed to eat only cookies, which required less self-regulatory effort (Segerstrom \& Nes, 2007). In addition, women with higher tonic HRV show higher phasic HRV while engaging in both emotion suppression and reappraisal, compared to those with lower tonic HRV (Butler et al., 2006). The authors suggest that higher phasic HRV during emotion regulation reflects greater attentional and emotional self-regulatory effort, and that women with higher tonic HRV exert greater emotional and attentional self-regulatory efforts while engaging in emotional regulation (Butler et al., 2006).

Taking the evidence together, whether phasic HRV suppression or enhancement reflects the exertion of self-regulatory effort may depend on the context in which phasic HRV changes occur. We construe phasic HRV suppression as an indicator of the exertion of self-regulatory effort when exposed to direct stress. Conversely, we construe phasic HRV enhancement as an indicator of the exertion of self-regulatory effort when performing a task that demands such efforts (e.g., employing emotion self-regulatory methods such as reappraisal, eating carrots, or inhibiting emotions to perform a goal-directed task). However, it is still unclear to what extent tonic cardiac vagal activity influences changes in phasic cardiac vagal activity under different levels of perceptual load. The current study is designed to further clarify the relationship between tonic and phasic cardiac vagal activity in a selective attentional task under different levels of perceptual load.

\section{The Load Theory of Selective Attention and Cognitive Control}

The physical world provides too much information, and the human capacity to process information is limited. Thus, the ability to focus on task-relevant stimuli and to ignore distractor stimuli-a phenomenon termed selective attention-is vital for successful selfregulation (Huang-Pollock, Carr, \& Nigg, 2002). For decades, researchers have debated whether selection takes place early or late in information processing (see Lavie, 1995). According to the early selection approach, human perception is limited, so selection takes place early, right after a rudimentary analysis of physical features, and only selected stimuli are fully perceived (Treisman, 1969). In contrast, the late selection approach proposes that selection occurs after full perception, which is unlimited and occurs in parallel fashion, but prevents distractors from influencing memory and behavior (termed distractor interference; Huang-Pollock et al., 2002; Norman, 1968). According to the load theory of selective attention and cognitive control (henceforth referred to as load theory), the locus of selection depends on perceptual load, or the total amount of potentially task-relevant information available (Lavie, 1995, 2005).

This theory argues that perception is limited (an "early" selection view), but all stimuli are automatically processed (a "late" selection view) as long as processing resources are available. Under low perceptual load, when the processing of task-relevant information is less demanding, there are more spare processing resources available to process task-irrelevant distractors. As a result, distractors significantly interfere with performance on goaldirected tasks. However, under high perceptual load, when the processing of task-relevant information is more demanding and complicated, there are fewer spare processing resources available to process task-irrelevant distractors (Lavie, Hirst, Fockert, \& Viding, 2004). As a result, distractors no longer interfere with the main task under high perceptual load. There is extensive evidence for load theory, such that task performance and neural activity are more likely to be affected by distractors under low relative to high perceptual load (see Lavie, 2005, for a review). Given the importance of perceptual load for successful self-regulation, we sought to understand whether tonic HRV could predict changes in phasic $\mathrm{HRV}$ in response to emotional distractors under different levels of load.

\section{Overview}

The current research examined the relationship between tonic cardiac vagal tone and changes in phasic cardiac activity to emotional stimuli. We predicted that individual differences in tonic 
HRV would predict phasic changes in HRV in response to emotionally negative versus neutral distractors under different levels of perceptual load. A recent study provides evidence that tonic HRV modulates phasic cardiac vagal activity in response to emotionally negative stimuli (Gaebler, Daniels, Lamke, Fydrich, \& Walter, 2013). Healthy controls, characterized by higher tonic HRV, showed phasic HRV enhancement in response to emotionally negative faces while completing an emotional face-matching task. Conversely, patients with social anxiety disorder, characterized by lower tonic HRV, showed phasic HRV suppression in response to emotionally negative faces. The results seem to suggest that people with higher tonic HRV may exert self-regulatory effort in response to emotionally negative stimuli, which allows them to perform the goal-directed task more effectively. In contrast, people with lower tonic HRV appear to exhibit a typical autonomic stress response to emotionally negative faces.

In the current study, participants were instructed to detect a target letter among letter strings superimposed either on fearful or neutral distractor faces (see Park, Vasey et al., 2013). Specifically, we hypothesized that (a) higher tonic HRV would be associated with significantly higher phasic HRV to fearful, compared to neutral, face distractors under low load (Butler et al., 2006). Significant phasic HRV enhancement observed in those with higher tonic HRV would suggest greater exertion of self-regulatory effort in response to fearful face distractors to support the execution of a goal-directed task more effectively. Indeed, we previously found that people with higher tonic HRV showed better task performance with fearful face distractors compared to neutral face distractors under low load (Park, Vasey et al., 2013). We also hypothesized that (b) low tonic HRV would be associated with phasic HRV suppression to fearful face distractors under low load-when more processing resources would be available to process distractors. There is no reason to activate the stress system for such trivial threat cues. However, people with lower tonic HRV may respond as if they are actually encountering a stressor triggering an autonomic stress response (i.e., phasic HRV suppression) in response to emotionally negative faces. Finally, we hypothesized that (c) tonic HRV would not be associated with changes in phasic HRV under high load, when fewer resources are available to exert self-regulatory effort or to process taskirrelevant distractors.

\section{Method}

\section{Participants}

Seventy-seven undergraduate students participated in the study for partial course credit. Participants were asked to refrain from smoking or drinking caffeinated beverages for $4 \mathrm{~h}$ before the experiment (Hansen et al., 2003). All participants had normal or corrected-to-normal vision (20/20 visual acuity). People with a history of vision disorders or dysfunctions, neurological or psychiatric disorders, cardiovascular disorders, or medical conditions such as diabetes were excluded from this experiment. Written informed consent was obtained from each participant before the experiment, and all procedures were approved by The Ohio State University Institutional Review Board. The behavioral and cardiovascular data from two participants were lost due to a computer error. We also excluded two participants who had more than $15 \%$ of missing trials due to errors and outliers, which yielded 73 participants (42 females; mean age $=20$; mean baseline $\mathrm{HRV}=6.57)$.

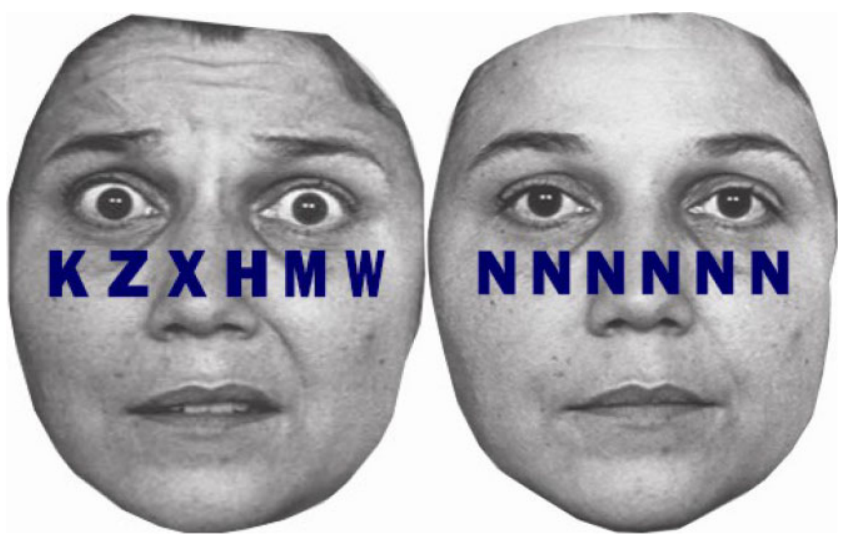

Figure 1. Example stimuli. A string of six letters was superimposed on fearful and neutral facial emotions. In the high cognitive load condition, letter strings consisted of one target letter and five nontarget letters $(\mathrm{H}, \mathrm{K}$, $\mathrm{M}, \mathrm{W}$, or Z) arranged in random order (left). In the low cognitive load condition, the letter string consisted of six Xs or six Ns (right).

\section{Stimuli}

Each display consisted of a face with the middle of the nose at fixation and a string of six letters, written in blue, superimposed across this middle point (see Figure 1). Fearful and neutral facial expressions of 12 different individuals (six women and six men) were selected from the Pictures of Facial Affect (Ekman \& Friesen, 1976). In the high cognitive load condition, the letter string comprised one target letter (X or $\mathrm{N}$ ) and five nontarget letters (H, K, M, $\mathrm{W}$, or $\mathrm{Z}$ ) arranged in random order. In the low cognitive load condition, the letter string consisted of six Xs or six Ns. Viewing distance was approximately $60 \mathrm{~cm}$. The particular faces used for the high and low load conditions were counterbalanced across participants (see Park, Vasey et al., 2013 for more details).

\section{Procedure}

All participants were tested individually in a dimly lit room. They were brought to the lab, and three surface electrodes were attached to obtain electrocardiographic (ECG) data. After placement of electrodes, tonic HRV was recorded for $5 \mathrm{~min}$, during which participants sat and rested quietly in a sound-isolated room. Participants then performed the letter detection task for $5 \mathrm{~min}$, during which task HRV was recorded. Four conditions crossing two facial emotions and two cognitive load conditions were administered in a between-subjects design.

The letter detection task. In the letter detection task, participants were presented with a series of letter strings and were instructed to identify whether each letter string contained an $\mathrm{X}$ or an $\mathrm{N}$ by pressing the corresponding keys on the keyboard as quickly and accurately as possible. Each block started with 12 practice trials with just the letter strings presented, followed by 48 experimental trials in random order. Each experimental trial began with a fixation cross for $500 \mathrm{~ms}$, followed by the display of the string of six letters superimposed on a face for $200 \mathrm{~ms}$. The interstimulus intervals were randomly generated with a mean of 4,500 ms (see Figure 2). Participants were told to ignore the faces. When participants failed to respond, they received feedback indicating that they had not responded in time. After the task, participants went through a 5-min recovery period. 


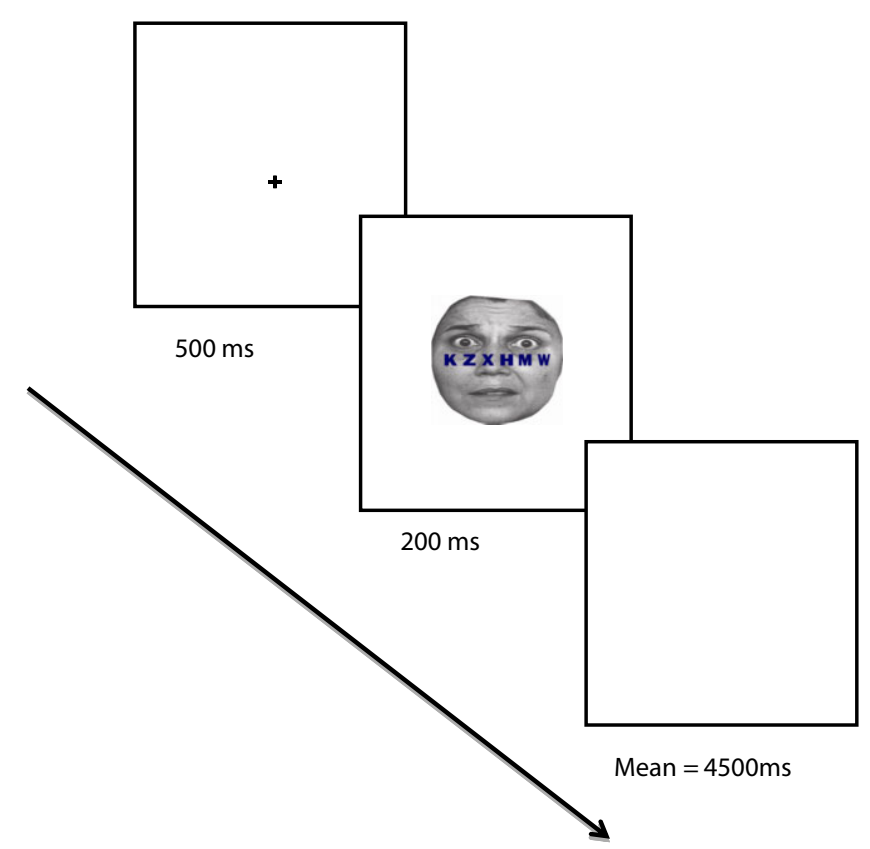

Figure 2. Example of experimental sequence. The fixation cross was presented for $500 \mathrm{~ms}$ and was followed by the display with a string of six letters superimposed on a face for $200 \mathrm{~ms}$. The interstimulus interval was randomly generated with a mean of $4,500 \mathrm{~ms}$. Stimuli are not drawn to scale.

\section{Physiological Measurements}

We recorded ECG activity via a standard three-electrode setup. The ECG signals, which were sampled at $1000 \mathrm{~Hz}$ (Task Force, 1996), passed through Mindware Technology's BioNex 50-3711-02 twoslot mainframe to an Optiplex GX620 personal computer (Pentium D, $2.80 \mathrm{GHz}, 2.00 \mathrm{~GB}$ RAM) running Mindware Technology's BioLab 1.11 software, which received digital triggers (100-ms pulses) via a parallel port connection with a second Optiplex GX620 running E-Prime 1.1.4.1 (Psychology Software Tools, Inc.). The ECG signals were inspected offline using Mindware Technology's HRV 2.51 software with which the ECG trace (plotted in $\mathrm{mV}$ against time) was carefully reexamined. Successive $\mathrm{R}$ spikes (identified by an automatic beat detection algorithm) were visually inspected, and any irregularities were edited. Successive interbeat intervals (in milliseconds) within the baseline and task performance period were written to a single text file. Time and frequency domain indices of the heart period power spectrum were analyzed and performed using the Kubios HRV analysis package 2.0 (http://kubios.uef.fi/). The Kubios software provides spectral estimates based upon the more modern autoregressive algorithm, which has numerous advantages over the fast Fourier transformbased algorithms (see Thayer, Hansen, \& Johnsen, 2008). We obtained high frequency HRV power that primarily reflects vagal influences using autoregressive estimates (Thayer \& Friedman, 2004; Thayer \& Ruiz-Padial, 2006). Spectral estimates of high frequency power (in milliseconds squared per hertz) were transformed logarithmically (base 10) to normalize the distribution (Ruiz-Padial, Sollers, Vila, \& Thayer, 2003). Log-transformed high frequency HRV power during baseline represented tonic HRV, and log-transformed high frequency HRV power during task performance represented task HRV.

\section{Analyses}

To assess the relationship between tonic HRV and phasic HRV during task performance under high and low perceptual load with fearful and neutral distractors, we conducted a 2 (Facial Emotion: fearful, neutral) $\times 2$ (Perceptual Load: high, low $) \times$ continuous (tonic HRV) multiple regression analysis on log high frequency task (phasic) HRV. Separate effect-coded variables were created for facial emotion (fear $=1$, neutral $=-1$ ) and perceptual load (high $=1$, low $=-1$ ). Log-transformed high frequency tonic and task HRV were $z$ standardized, and interaction terms were computed from these standardized scores (Aiken \& West, 1991).

\section{Results}

The regression analysis yielded several significant effects. First, there was a main effect of HRV, $\beta=.21, t(66)=3.21, p<.01$, $f^{2}=2.64$, such that higher tonic HRV was associated with higher phasic HRV during the task. Second, there was a significant twoway interaction between facial emotion and perceptual load, $\beta=.12, t(66)=2.04, p<.05, f^{2}=.26 .{ }^{1}$ However, simple slopes analyses (Aiken \& West 1991; Preacher, Curran, \& Bauer, 2006) showed that there was no association between facial emotion and task HRV in either the high or low load conditions ( $p s>.43$ ). Finally, there was a significant two-way interaction between facial emotion and HRV, $\beta=.21, t(66)=3.21, p<.01, f^{2}=.26 .^{2}$ Consistent with our predictions, simple slopes analyses (Aiken \& West, 1991; Preacher et al., 2006) showed that higher tonic HRV was associated with a significant increase in phasic HRV from baseline during the conditions with fearful face distractors compared to the conditions with neutral face distractors, regardless of the level of perceptual load, $\beta=.19, t(70)=2.08, p<.04$. In contrast, lower tonic HRV was associated with a marginally significant decrease in phasic HRV, regardless of perceptual load, $\beta=-.16, t(70)=-1.70$, $p=.10$. In other words, higher tonic HRV was associated with a significant increase in phasic HRV from baseline during the conditions with fearful face distractors, whereas lower tonic HRV was associated with a marginally significant decrease in phasic HRV.

We had hypothesized that (a) high tonic HRV would be associated with phasic HRV enhancement in the conditions with fearful face distractors under low perceptual load, (b) low tonic HRV would be associated with phasic HRV suppression on the condition with fearful face distractors under low load, and (c) tonic HRV would not be associated with phasic HRV under high load when

1. In addition, there was a marginally significant two-way interaction between perceptual load and HRV, $\beta=.23, t(66)=1.80, p<.08, f^{2}=.26$. However, simple slopes analyses (Aiken \& West, 1991; Preacher et al., 2006) showed that there was no effect of tonic HRV on perceptual load $(p>.48)$. When we conducted three-way multiple regression analyses on mean heart rate and respiration examined by the high frequency peak (hz), there was no significant interaction $(p>1.42)$.

2. We also examined the relationship between tonic HRV and HRV reactivity (e.g., phasic HRV during the task minus tonic HRV). Consistent with the relationship between tonic HRV and phasic HRV, there was a significant two-way interaction between facial emotion and HRV, $\beta=.40$, $t(66)=3.21, p<.01, f^{2}=.26$. Simple slopes analyses (Aiken \& West, 1991; Preacher et al., 2006) showed that higher tonic HRV predicted a significant greater HRV reactivity during the conditions with fearful faces compared to the conditions with neutral faces, $\beta=.35, t(70)=2.20, p<.04$. In contrast, lower tonic HRV was marginally related to reduced HRV reactivity during the conditions with fearful faces compared to the conditions with neutral faces, $\beta=-.29, t(70)=-1.79, p=.08$. Thus, high tonic HRV was associated with significantly greater HRV reactivity during the conditions with fearful distractors, whereas low tonic HRV was not. 


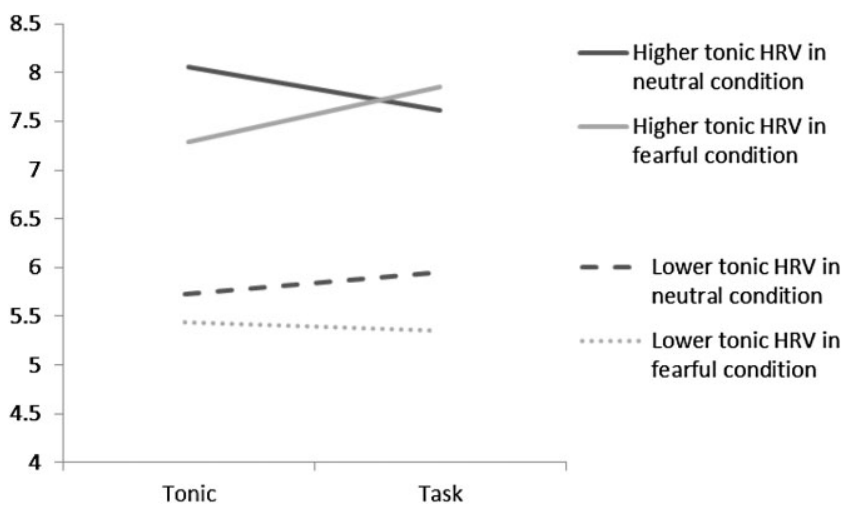

Figure 3. Log-transformed high frequency power heart rate variability (HRV) during tonic and task performance as a function of high and low tonic HRV levels and facial emotions under low load. Participants were divided into two groups - high or low HRV — based on the median split of log-transformed high frequency HRV during baseline (median $=6.57$ ) for graphic purposes only.

fewer resources were available. Therefore, although the three-way interaction between perceptual load, facial emotion, and HRV was not significant $(p=.16)$, we examined the interaction between facial emotion and HRV under low load and under high load separately in order to directly examine these hypotheses. As expected, there was a significant two-way interaction between facial emotion and tonic HRV under low load, $\beta=.28, t(34)=3.06$, $p<.01, f^{2}=.27$ (see Figure 3 ). ${ }^{3}$ In contrast, this interaction was not statistically significant under high load, $\beta=.13, t(31)=1.52$, $p=.14$ (see Figure 4). As predicted, simple slopes analyses (Aiken \& West, 1991; Preacher et al., 2006) revealed that higher tonic HRV was associated with an increase in phasic HRV from baseline during the conditions with fearful face distractors compared to the conditions with neutral face distractors, $\beta=.46, t(34)=10.29$, $p<.001$. Conversely, lower tonic HRV was associated with a

3. Consistent with the relationship between tonic HRV and phasic HRV, there was a significant two-way interaction between facial emotion and HRV reactivity under low load, $\beta=.48, t(34)=3.06, p<.01, f^{2}=.28$, but not under high load $(p=.14)$.

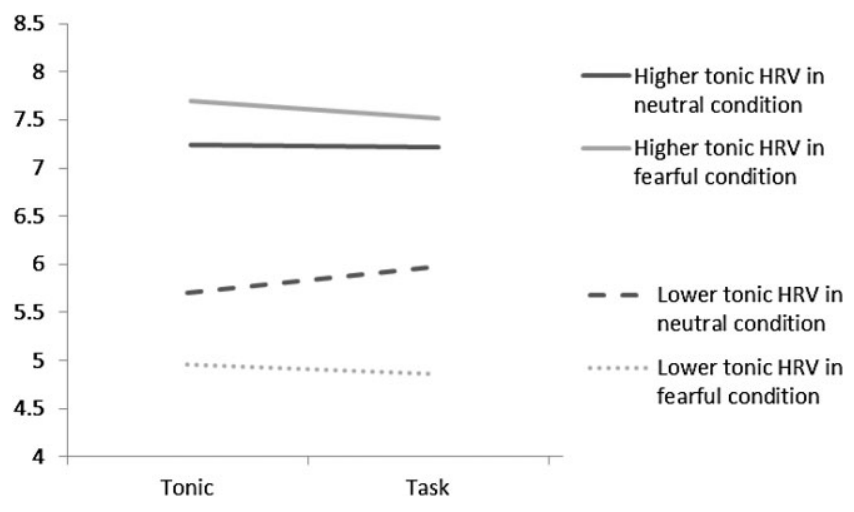

Figure 4. Log-transformed high frequency power HRV during tonic and task performance as a function of high and low tonic HRV levels and facial emotions under high load. Participants were divided into two groups-high or low HRV - based on the median split of log-transformed high frequency HRV during baseline (median $=6.57$ ) for graphic purposes only.

decrease in phasic HRV from baseline during the conditions with fearful face distractors compared to the conditions with neutral face distractors, $\beta=-.16, t(34)=-3.58, p<.01$ (see Table 1 and 2 for dichotomous data based on the median split of tonic HRV). Under high load, simple slopes analyses revealed that higher tonic HRV was associated with a nonsignificant increase in phasic HRV, $\beta=.11, t(30)=0.27, p=.79$, whereas lower tonic HRV was associated with a significant decrease in phasic HRV from baseline during the condition with fearful distractors, $\beta=-.21$, $t(30)=-2.30, p<.01$. Thus, in both the high load and low load conditions, participants with lower tonic HRV showed significant phasic HRV suppression in conditions with fearful distractors. Importantly, those with higher tonic HRV showed significant phasic HRV enhancement under low load and no evidence of HRV suppression-even under high load.

\section{Discussion}

In the current research, we examined whether tonic cardiac vagal activity would predict changes in phasic cardiac vagal activity in

Table 1. Means and Standard Deviations (SD) of High Frequency Heart Rate Variability (HRV) Power in People with Higher Tonic HRV

\begin{tabular}{|c|c|c|c|c|c|c|c|}
\hline & \multicolumn{3}{|c|}{ High perceptual load } & & \multicolumn{3}{|c|}{ Low perceptual load } \\
\hline & Baseline & Task & $\begin{array}{c}\text { Phasic } \\
\text { HRV }\end{array}$ & & Baseline & Task & $\begin{array}{c}\text { Phasic } \\
\text { HRV }\end{array}$ \\
\hline \multicolumn{8}{|l|}{ Fearful faces } \\
\hline HRV & 7.7 & 7.5 & -.18 & HRV & 7.3 & 7.9 & -.02 \\
\hline$S D$ & .8 & .9 & .5 & $S D$ & .7 & 2.2 & .33 \\
\hline Heart rate & 64.4 & 65.6 & & Heart rate & 67.8 & 67.5 & \\
\hline$S D$ & 8.8 & 9.2 & & $S D$ & 10.7 & 9.6 & \\
\hline Respiration & .24 & .26 & & Respiration & .28 & .28 & \\
\hline$S D$ & .05 & .07 & & $S D$ & .05 & .06 & \\
\hline \multicolumn{8}{|l|}{ Neutral faces } \\
\hline HRV & 7.2 & 7.2 & .56 .13 & HRV & 8.1 & 7.6 & -0.44 \\
\hline$S D$ & .3 & .4 & 1.7 & $S D$ & 1.1 & 1.1 & .58 \\
\hline Heart rate & 68.1 & 67.2 & & Heart rate & 69.7 & 71.6 & \\
\hline$S D$ & 10.4 & 9.1 & & $S D$ & 7.5 & 9.0 & \\
\hline Respiration & .29 & .27 & & Respiration & .24 & .26 & \\
\hline$S D$ & .04 & 0.03 & & $S D$ & .09 & .03 & \\
\hline
\end{tabular}

Note. Phasic HRV is estimated by contrasting HRV during the task with HRV during the baseline. To categorize participants into higher tonic HRV group, we used the median split of log-transformed high frequency HRV during baseline (median $=6.57$ ). 
Table 2. Means and Standard Deviations (SD) of High Frequency Heart Rate Variability (HRV) Power in People with Lower Tonic HRV

\begin{tabular}{|c|c|c|c|c|c|c|c|}
\hline & \multicolumn{3}{|c|}{ High perceptual load } & & \multicolumn{3}{|c|}{ Low perceptual load } \\
\hline & Baseline & Task & $\begin{array}{c}\text { Phasic } \\
\text { HRV }\end{array}$ & & Baseline & Task & $\begin{array}{c}\text { Phasic } \\
\text { HRV }\end{array}$ \\
\hline \multicolumn{8}{|l|}{ Fearful faces } \\
\hline HRV & 5.2 & 5.0 & -.18 & HRV & 5.4 & 5.4 & .04 \\
\hline$S D$ & 1.1 & 1.2 & .38 & $S D$ & .5 & .7 & .58 \\
\hline Heart rate & 87.9 & 87.8 & & Heart rate & 88.1 & 87.4 & \\
\hline$S D$ & 12.4 & 11.8 & & $S D$ & 10.7 & 11.1 & \\
\hline Respiration & .28 & .31 & & Respiration & .26 & .29 & \\
\hline$S D^{1}$ & .04 & .05 & & $S D$ & .03 & .03 & \\
\hline \multicolumn{8}{|l|}{ Neutral faces } \\
\hline HRV & 5.7 & 6.0 & .26 & HRV & 5.7 & 5.9 & .22 \\
\hline$S D$ & .7 & .9 & .93 & $S D$ & .5 & .7 & .33 \\
\hline Heart rate & 79.4 & 78.5 & & Heart rate & 82.7 & 81.4 & \\
\hline$S D$ & 7.7 & 9.2 & & $S D$ & 8.8 & 9.7 & \\
\hline Respiration & .29 & .31 & & Respiration & .26 & .28 & \\
\hline$S D$ & .04 & .05 & & $S D$ & .03 & .04 & \\
\hline
\end{tabular}

Note. Phasic HRV is estimated by contrasting HRV during the task with HRV during the baseline. To categorize participants into lower tonic HRV group, we used the median split of log-transformed high frequency HRV during baseline ( $\operatorname{median}=6.57$ ).

response to emotionally negative distractors. As predicted, when processing resources were available, higher tonic HRV was associated with phasic HRV enhancement, whereas lower tonic HRV was associated with phasic HRV suppression, in response to fearful distractors. However, contrary to our prediction, when processes resources were limited in the high load condition, those with lower tonic HRV still showed phasic HRV suppression to fearful distractors, whereas those with higher tonic HRV showed no significant change in phasic HRV. These findings suggest that people with higher tonic HRV show phasic HRV enhancement, which can be construed as an indicator of the exertion of self-regulatory effort, whereas people with lower tonic HRV show phasic HRV suppression, which can be construed as an indicator of the autonomic stress response to emotionally negative stimuli.

Our results extend previous research in a number of ways. First, our research suggests that phasic HRV enhancement and suppression associated with higher and lower tonic HRV, respectively, occur when processing resources are available under low load. However, when processing resources are limited, people with low tonic HRV still appear to process the fearful distractors based upon their phasic HRV decrease. Furthermore, we have previously shown that those with higher tonic HRV perform better on the primary task relative to those with lower tonic HRV (Park, Vasey et al. 2013). Taken together, these results suggest that people with low tonic HRV may divert resources from goal-directed task performance to process potential threats in the environment, whereas those with higher tonic HRV are better able to inhibit taskirrelevant distractors in order to successfully perform a task.

Our results are consistent with previous research reporting that people who are characterized by lower tonic HRV showed phasic HRV suppression, but people who are characterized by higher tonic HRV showed phasic HRV enhancement in response to emotionally negative facial stimuli while performing an emotional facematching task (Aldao \& Mennin, 2012; Gaebler et al., 2013). Similarly, another study reports that people with higher tonic HRV show phasic HRV enhancement, coupled with more positive affect, but people with lower tonic HRV show phasic suppression, coupled with less positive affect, during the recall of a stressful event (Cribbet, Williams, Gunn, \& Rau, 2011). However, our results further clarify these findings by showing that perceptual load mod- erates the relationship between tonic HRV and phasic HRV differentially in persons that differ on tonic HRV.

The current research contributes to a growing body of research showing that higher tonic cardiac vagal tone is associated with flexible regulatory function (Appelhans \& Luecken, 2006; Lane et al., 2013; Porges, 1991, 2003; Thayer \& Lane, 2000, 2009). In other words, people with higher tonic HRV have the capacity to exert self-regulatory effort to respond more adaptively to momentto-moment situational changes, which allows them to perform a goal-relevant task more effectively. Indeed, people with higher tonic HRV perform better in a condition with fearful distractors compared to neutral distractors under low load (Park, Vasey et al., 2013). The exertion of flexible self-regulatory effort also contributes to positive physical and psychological outcomes (Appelhans \& Luecken, 2006; Lane et al., 2013; Porges, 1991, 2003; Thayer \& Lane, 2000, 2009). Higher tonic HRV has been frequently linked with positive emotions and social well-being (Kok \& Fredrickson, 2010). Conversely, people with lower tonic HRV appear to produce an autonomic stress response to fearful distractors. Although there is no reason to activate the autonomic stress system for such trivial threat cues, people with lower tonic HRV may respond to them as if these trivial cues are significant stressors. The autonomic stress response can activate a cascade of physiological defensive systems, which eventually facilitate physical wear and tear of the systems. Not surprisingly, lower tonic HRV has been associated with physiological problems, such as hypertension, diabetes, high cholesterol, obesity, arthritis, and some cancers (Friedman \& Thayer, 1998; Park, Van Bavel, Vasey, \& Thayer, 2013; Thayer et al., 1996).

It should be noted that behavioral data from our previous paper (Park, Vasey et al., 2013) confirmed that our load manipulation was successful. High perceptual load resulted in significantly slower reaction times and more mean errors than the low perceptual load. Results of that study extended load theory, showing that individual differences in HRV can modulate task performance under high load. People with high tonic HRV were faster in the condition with neutral face distractors, but not with fearful face distractors, under high load; however, people with low tonic HRV were slower in the conditions with both fearful and neutral face distractors. It appears that people with higher tonic HRV may have recognized neutral distractors as "safety cues" under high load, which raises a 
possibility that task-irrelevant neutral distractors may have been processed to some extent under high load. However, it is difficult to capture the corresponding physiological response because most physiological responses are more sensitive to fearful compared to neutral stimuli.

On the other hand, the current study examining the relationship between tonic and phasic HRV is also consistent with aspects of load theory. Resting HRV predicted phasic cardiac responses to fearful distractors under low load, whereas the modulation of tonic HRV on phasic cardiac activity was less apparent under high load. In the presence of fearful face distractors, people with both high and low HRV may experience difficulty mobilizing and recruiting resources to respond under high load. Importantly, people with low tonic HRV showed phasic HRV suppression even under high load, suggesting that they allocated resources to process threat as evidenced by an autonomic stress response to fearful face distractors.

It is possible that people with higher tonic HRV may have engaged in involuntary, automatic self-regulation ("weak" automaticity; Okon-Singer, Lichtenstein-Vidne, \& Cohen, 2013) in the condition with fearful face distractors under high load. Automaticity has been defined as being "weak" and "strong" (Okon-Singer et al., 2013). Strong automaticity has been referred to as being completely independent of top-down influences, such as attention and task demands, whereas weak automaticity as being taskirrelevant or involuntary. We defined automatic self-regulatory effort under high load as weak because task performance (reaction time) was affected by task load (Okon-Singer et al., 2013; Park, Vasey et al., 2013). However, persons with higher tonic HRV may have engaged in relatively "automatic" as well as more "controlled" components of self-regulation under low load when more processing resources were available (see also Cunningham, Zelazo, Packer, \& Van Bavel, 2007). It is possible that people with higher tonic HRV may have exerted different types of self-regulatory effort depending on the availability of processing resources; however, it is yet to be determined in future research.

There are some limitations. In the current study, we used a between-subjects design to minimize practice effects (Park, Vasey et al., 2013). Therefore, the effect of individual differences in phasic HRV in response to fearful versus neutral face distractors under high and low load may be even clearer using a within-subject design.

\section{Conclusion}

The current study provides additional evidence that individual differences in tonic cardiac vagal tone are associated with changes in phasic cardiac vagal activity in response to emotionally distracting situations. Moreover, these changes depend on the availability of perceptual resources. As such, tonic cardiac vagal tone-a correlate of self-regulatory functioning - is associated with the capacity to make autonomic responses that are more adaptive to moment-tomoment situational changes when processing resources are available.

\section{References}

Aldao, A., \& Mennin, D. S. (2012). Paradoxical cardiovascular effects of implementing adaptive emotion regulation strategies in generalized anxiety disorder. Behaviour Research \& Therapy, 50, 122-130.

Aiken, L. S., \& West, S. G. (1991). Multiple regression: Testing and interpreting interactions. Newbury Park, CA: Sage.

Appelhans, B. M., \& Luecken, L. J. (2006). Heart rate variability as an index of regulated emotional responding. Review of General Psychology, 10, 229-240. doi: 10.1037/1089-2680.10.3.229

Beauchaine, T. P., Gatzke-Kopp, L., \& Mead, H. K. (2007). Polyvagal theory and development psychopathology: Emotion dysregulation and conduct problems from preschool to adolescence. Biological Psychology, 74, 174-184. doi: 10.1016/j.biopsycho.2005.08.008

Beauchaine, T. P., Katkin, E. S., Strassberg, Z., \& Snarr, J. (2001). Disinhibitory psychopathology in male adolescents: Discriminating conduct disorder from attention-deficit/hyperactivity disorder through concurrent assessment of multiple autonomic states. Journal of Abnormal Psychology, 110, 610-624. doi: 10.1037/0021-843X.110.4.610

Berntson, G. G., Bigger, J. T., Eckberg, D. L., Grossman, P., Kaufmann, P. G., Malik, M., . . . Van Der Molen, M. W. (1997). Heart rate variability: Origins, methods, and interpretive caveats. Psychophysiology, 34, 623-648.

Brosschot, J. F., Van Dijk, E., \& Thayer, J. F. (2007). Daily worry is related to low heart rate variability during waking and the subsequent nocturnal sleep period. International Journal of Psychophysiology, 63, 39-47.

Butler, E. A., Wilhelm, F. H., \& Gross, J. J. (2006). Respiratory sinus arrhythmia, emotion, and emotion regulation during social interaction. Psychophysiology, 43, 612-622. doi: 10.1111/j.1469-8986.2006. 00467.x

Cribbet, M. R., Williams, P. G., Gunn, H. E., \& Rau, H. K. (2011). Effects of tonic and phasic respiratory sinus arrhythmia on affective stress responses. Emotion, 11, 188-193. doi: 10.1037/a0021789

Cunningham, W. A., Zelazo, P. D., Packer, D. J., \& Van Bavel, J. J. (2007). The iterative reprocessing model: A multi-level framework for attitudes and evaluation. Social Cognition, 25, 736-760. doi: 10.1521/soco. 2007.25.5.736

Eisenberg, N. (2001). The core and correlates of affective social competence. Social Development, 10, 120-124. doi: 10.1111/1467-9507. 00151
Ekman, P., \& Friesen, W. V. (1976). Pictures of facial affect. Palo Alto, CA: Consulting Psychologists Press.

El-Sheikh, M., Hinnant, J. B., \& Erath, S. (2011). Developmental trajectories of delinquency symptoms in childhood: The role of marital conflict and autonomic nervous system activity. Journal of Abnormal Psychology, 120, 16-32. doi: 10.1037/0012-1649.39.2.222

Friedman, B. H., \& Thayer, J. F. (1998). Autonomic balance revisited: Panic anxiety and heart rate variability. Journal of Psychosomatic Research, 44, 133-151.

Fabes, R. A., \& Eisenberg, N. (1997). Regulatory control and adults' stress-related responses to daily life events. Journal of Personality and Social Psychology, 73, 1107-1117. doi: 10.1037/0022-3514.73.5.1107

Funder, D. C., \& Block, J. (1989). The role of ego-control, ego-resiliency, and IQ in delay of gratification in adolescence. Journal of Personality and Social Psychology, 57, 1041-1050. doi: 10.1037/0022-3514.57. 6.1041

Funder, D. C., Block, J. H., \& Block, J. (1983). Delay of gratification: Some longitudinal personality correlates. Journal of Personality and Social Psychology, 44, 1198-1213. doi: 10.1037/0022-3514.44.6.1198

Gaebler, M., Daniels, J. K., Lamke, J.-P., Fydrich, T., \& Walter, H. (2013). Heart rate variability and its neural correlates during emotional face processing in social anxiety disorder. Biological Psychology. 94, 319330 .

Gottfredson, M., \& Hirschi, T. (1990). A general theory of crime. Stanford, CA: Stanford University Press.

Gross, J. J. (1998). Antecedent- and response-focused emotion regulation: Divergent consequences for experience, expression, and physiology. Journal of Personality and Social Psychology, 74, 224-237. doi: 10.1037/0022-3514.74.1.224

Hansen, A. L., Johnsen, B. H., \& Thayer, J. F. (2003). Vagal influence on working memory and attention. International Journal of Psychophysiology, 48, 263-274. doi: 10.1016/S0167-8760(03)00073-4

Huang-Pollock, C. L., Carr, T. H., \& Nigg, J. T. (2002). Development of selective attention: Perceptual load influences early versus late attentional selection in children and adults. Developmental Psychology, 38, 363-375. doi: 10.1037/0012-1649.38.3.363

Ingjaldsson, J. T., Laberg, J. C., \& Thayer, J. F. (2003). Reduced heart rate variability in chronic alcohol abuse: Relationship with negative mood, 
chronic thought suppression, and compulsive drinking. Biological Psychiatry, 54, 1427-1436. doi: 10.1016/S0006-3223(02)01926-1

Kok, B. E., \& Fredrickson, B. L. (2010). Upward spirals of the heart: Autonomic flexibility, as indexed by vagal tone, reciprocally and prospectively predicts positive emotions and social connectedness. Biological Psychology, 83, 432-436. doi: 10.1016/j.biopsycho.2010.09.005

Lane, R. D., Weidenbacher, H., Smith R., Fort, C., Thayer, J. F., \& Allen, J. J. B. (2013). Subgenual anterior cingulate cortex activity covariation with cardiac vagal control is altered in depression. Journal of Affective Disorders, 100, 103-119. doi: 10.1037/a0021136

Lavie, N. (1995). Perceptual load as a necessary condition for selective attention. Journal of Experimental Psychology: Human Perception and Performance. 21, 451-468. doi: 10.1037/0096-1523.21.3.451

Lavie, N. (2005). Distracted and confused?: Selective attention under load. Trends in Cognitive Science, 9, 75-82. doi: 10.1016/j.tics.2004.12.004

Lavie, N., Hirst, A., Fockert, J. W. D., \& Viding, E. (2004). Load theory of selective attention and cognitive control. Journal of Experimental Psychology: General, 133, 339-354. doi: 10.1037/0096-3445.133.3.339

Lyonfields, J. D., Borkovec, T. D., \& Thayer, J. F. (1995). Vagal tone in generalized anxiety disorder and the effects of aversive imagery and worrisome thinking. Behavior Therapy, 26, 457-466.

Martin, L. L., \& Tesser, A. (1989). Towards a motivational and structural theory of ruminative thought. In J. S. Uleman \& J. A. Bargh (Eds.), Unintended thought (pp. 306-326). New York, NY: Guilford Press.

McCullough, M. E., \& Willoughby, B. L. (2009). Religion, self-regulation, and self-control: Associations, explanations, and implications. Psychological Bulletin, 135, 69-93. doi: 10.1037/a0014213

Norman, D. A. (1968). Toward a theory of memory and attention. Psychological Review, 75, 522-536. doi: 10.1037/h0026699

Okon-Singer, H., Lichtenstein-Vidne, L., \& Cohen, N. (2013). Dynamic modulation of emotional processing. Biological Psychology, 92, 480491.

Oveis, C., Cohen, A. B., Gruber, J., Shiota, M. N., Haidt, J., \& Keltner, D. (2009). Resting respiratory sinus arrhythmia is associated with tonic positive emotionality. Emotion, 9, 265-270. doi: 10.1037/a0015383

Park, G., Van Bavel, J. J., Vasey, M. W., \& Thayer, J. F. (2012). Cardiac vagal tone predicts inhibited attention to fearful faces. Emotion, 12, 1292-1302. doi: $10.1037 / \mathrm{a} 0028528$

Park, G., Van Bavel, J. J., Vasey, M., \& Thayer, J. F. (2013). Cardiac vagal tone predicts attentional engagement to and disengagement from fearful faces. Emotion, 13, 645-656. doi: 10.1037/a0032971

Park, G., Vasey, M., Van Bavel, J. J., \& Thayer, J. F. (2013). Cardiac vagal tone is correlated with selective attention to neutral distractors under load. Psychophysiology, 50, 398-406. doi: 10.1111/psyp.12029

Porges, S. W. (1991). Vagal tone: An autonomic mediator of affect. In J. A. Garber \& K. A. Dodge (Eds.), The development of affect regulation and dysregulation (pp. 11-128). New York, NY: Cambridge University Press.

Porges, S. W. (2003). The polyvagal theory: Phylogenetic contributions to social behavior. Physiology \& Behavior, 79, 503-513. doi: 10.1016/ S0031-9384(03)00156-2

Preacher, K. J., Curran, P. J. \& Bauer, D. J. (2006). Computational tools for probing interactions in multiple linear regression, multilevel modeling, and latent curve analysis. Journal of Educational and Behavioral Statistics, 31, 437-448. doi: 10.3102/10769986031004437

Pyszczynski, T., Holt, K., \& Greenberg, J. (1987). Depression, self-focused attention, and expectancies for positive and negative future life events for self and others. Journal of Personality and Social Psychology, 52, 994-1001. doi: 10.1037//0022-3514.52.5.994

Ruiz-Padial, E., Sollers, J. J., Vila, J., \& Thayer, J. F. (2003). The rhythm of the heart in the blink of an eye: Emotion-modulated startle magnitude covaries with heart rate variability. Psychophysiology, 40, 306-313. doi: $10.1111 / 1469-8986.00032$

Schwerdtfeger, A., \& Derakhshan, N. (2010). The time line of threat processing and vagal withdrawal in response to a self-threatening stressor in cognitive avoidant copers: Evidence for vigilance- avoidance theory. Psychophysiology, 47, 786-795. doi: 10.1111/ j.1469-8986.2010.00965.x

Segerstrom, S. C., \& Nes, L. S. (2007). Heart rate variability reflects self-regulatory strength, effort, and fatigue. Psychological Science, 18, 275-281. doi: 10.1111/j.1467-9280.2007.01888.x

Smith, T. W., Cribbet, M. R., Nealey-Moore, J. B., Uchino, B. N., Williams, P. G., MacKenzie, J., \& Thayer, J. F. (2011). Matters of the variable heart: Respiratory sinus arrhythmia response to marital interaction and associations with marital quality. Journal of Personality and Social Psychology, 100, 103-119. doi: 10.1037/a0021136

Task Force of the European Society of Cardiology and the North American Society of Pacing and Electrophysiology. (1996). Heart rate variability: Standards of measurement, physiology interpretation, and clinical use. Circulation, 93, 1043-1065. doi: 10.1161/01.CIR.93.5.1043

Thayer, J. F., \& Friedman, B. H. (2004). A neurovisceral integration model of health disparities in aging. In N. B. Anderson, R. A. Bulatao, \& B. Cohen (Eds.), Critical perspective on racial and ethnic differences in health in late life (pp. 567-603). Washington, DC: The National Academies.

Thayer, J. F., Friedman, B. H., \& Borkovec, T. D. (1996). Autonomic characteristics of generalized anxiety disorder and worry. Biological Psychiatry, 39, 255-266. doi: 10.1016/0006-3223(95)00136-0

Thayer, J. F., Hansen, A. L., \& Johnsen, B. H. (2008). Non-invasive assessment of autonomic influences on the heart: Impedance cardiography and heart rate variability. In L. J. Luecken \& L. C. Gallo (Eds.), Handbook of physiological research methods in health psychology (pp. 183-209). Newbury Park, CA: Sage Publications.

Thayer, J. F., Hansen, A. L., Saus-Rose, E., \& Johnsen, B. H. (2009). Heart rate variability, prefrontal neural function and cognitive performance: The neurovisceral integration perspective on self-regulation, adaptation, and health. Annals of Behavioral Medicine, 37, 141-153. doi: 10.1007/ s12160-009-9101-z

Thayer, J. F., \& Lane, R. D. (2000). A model of neurovisceral integration in emotion regulation and dysregulation. Journal of Affective Disorder, 61, 201-216. doi: 10.1016/S0165-0327(00)00338-4

Thayer, J. F., \& Lane, R. D. (2009). Claude Bernard and the heart-brain connection: Further elaboration of a model of neurovisceral integration. Neuroscience \& Biobehavioral Reviews, 33, 81-88. doi: 10.1016/ j.neubiorev.2008.08.004

Thayer, J. F., \& Ruiz-Padial, E. (2006). Neurovisceral integration, emotions and health: An update. International Congress Series, 1287, 122-127. doi: 10.1016/S0165-0327(00)00338-4

Tice, D. M., \& Baumeister, R. F. (1993). Controlling anger: Self-induced emotion change. In D. M. Wegner \& J. W. Pennebaker (Eds.), Handbook of mental control (pp. 393-409). Englewood Cliffs, NJ: Prentice Hall.

Treisman, A. M. (1969). Strategies and models of selective attention. Psychological Review, 76, 282-299. doi: 10.1037/h0027242

Uijtdehaage, S. H., \& Thayer, J. F. (2000). Accentuated antagonism in the control of human heart rate. Clinical Autonomic Research, 10, 107-110. doi: 10.1007/BF02278013

Weber, C. S., Thayer, J. F., Rudat, M., Wirtz, P. H., Zimmermann-Viehoff, F., Thomas, A., ... Deter, H. C. (2010). Low vagal tone is associated with impaired post stress recovery of cardiovascular, endocrine, and immune markers. European Journal of Applied Physiology, 109, 201211. doi: $10.1007 / \mathrm{s} 00421-009-1341-x$

Wegner, D. M., Schneider, D. J., Carter, S. R., \& White, T. L. (1987). Paradoxical effects of thought suppression. Journal of Personality and Social Psychology, 53, 5-13. doi: 10.1037/0022-3514.53.1.5

Wenzlaff, R. M., Wegner, D. M., \& Roper, D. W. (1988). Depression and mental control: The resurgence of unwanted negative thoughts. Journal of Personality and Social Psychology, 55, 882-892. doi: 10.1037/00223514.55.6.882

(ReCEIVed February 13, 2013; ACCEPTED November 13, 2013) 